\title{
A Decline in Glomerular Filtration Rate Rather than Renal Arterial Stenotic Lesions, per se, Predicts Cardiovascular-Renal Events in Type 2 Diabetic Patients
}

\author{
Susumu Ogawa, MD, PhD; Kazuhiro Nako, MD; Masashi Okamura, MD, PhD; Miho Senda, MD; \\ Takuya Sakamoto, MD; Takaaki Abe, MD, PhD; Sadayoshi Ito, MD, PhD
}

\begin{abstract}
Background: In diabetic patients with renal artery arteriosclerosis (RAAS), the factors associated with a greater risk for cardiovascular-renal events (CVREs) remain unclear: the decline in estimated glomerular filtration rate (eGFR) caused by RAAS or the advance of arteriosclerosis that causes RAAS. Hence, the features to determine which best predicts the onset of CVREs in such patients were compared.
\end{abstract}

\begin{abstract}
Methods and Results: The renal arteries of 162 type 2 diabetes patients were assessed by using magnetic resonance angiography (RAAS diagnosed as arteriosclerotic stenosis $\geq 50 \%$ ) and they were studied longitudinally over 7 years. The influence of the presence/absence of RAAS, a decline in eGFR, clinical factors, surrogate arteriosclerotic markers and ischemic markers on patient's CVREs were assessed. A Cox regression analysis showed the detection of RAAS to be an independent risk factor for CVREs (bilateral RAAS was an extremely strong risk factor for the development of CVREs within 1,000 days), as was the decline in eGFR in a logistic regression analysis; the latter being a more powerful risk factor for CVREs. A multiple regression analysis revealed angiopoietin-2, a marker of ischemia, to be a risk factor for the decline in eGFR.
\end{abstract}

Conclusions: A decline in renal function but not the renal arterial stenotic lesion itself appears to be associated with an increased incidence of CVREs in type 2 diabetic patients with RAAS. (Circ J 2013; 77: 2816-2822)

Key Words: Angiopoietin-2; Cardiovascular-renal event; Estimated glomerular filtration rate; Ischemic renal injury; Renal artery arteriosclerosis

$\mathbf{R}$ ecently, many cohort studies in Japan have confirmed that type 2 diabetes mellitus is associated with an increased risk of cardiovascular-renal events (CVREs). ${ }^{1}$ Renal artery arteriosclerosis (RAAS) in diabetes mellitus is believed to reflect systemic arteriosclerosis and, accordingly, it is likely to represent a risk factor for CVREs. Such RAAS might indicate that damage to more peripheral microcirculation in the kidney has already progressed to some degree, as reflected in the decline in the estimated glomerular filtration rate (eGFR), leading to an increased incidence of CVREs. Thus, both RAAS and the decline in eGFR appear to be risk factors for CVREs in diabetic patients with RAAS, and systemic and/or local ischemia might be involved in these pathologies.

\section{Editorial p 2700}

These facts prompted us to conduct a longitudinal study to clarify whether, in diabetic patients, RAAS and/or a decline in eGFR is a risk factor for CVREs and which of these features is the more powerful independent risk factor for CVREs, and whether angiopoietin-2, a marker of ischemia, is a risk factor for CVREs. In addition, due to their potential involvement in arteriosclerosis and renal damage, increasing attention is being paid to substances in the blood and urine as predictors of CVREs (such as inflammatory cytokines, chemokines and oxidative stress markers). ${ }^{2}$ However, because the relationship of these factors with RAAS remains unknown, we investigated the association between these substances and RAAS in this 7-year longitudinal cohort study, as well as that between RAAS and CVREs.

Received February 26, 2013; revised manuscript received May 9, 2013; accepted June 11, 2013; released online August 6, 2013 Time for primary review: 29 days

Division of Nephrology, Endocrinology and Vascular Medicine, Tohoku University Hospital, Sendai (S.O., K.N., M.O., M.S., T.S., S.I.); Center for the Advancement of Higher Education, Tohoku University, Sendai (S.O.); Division of Medical Science, Tohoku University Graduate School of Biomedical Engineering, Sendai (T.A.); and Department of Clinical Biology and Hormonal Regulation, Tohoku University Graduate School of Medicine, Sendai (T.A.), Japan

Mailing address: Susumu Ogawa MD, PhD, Division of Nephrology, Endocrinology and Vascular Medicine, Tohoku University Hospital, 1-1 Seiryo-machi, Aoba-ku, Sendai 980-8574, Japan. E-mail: ogawa-s@hosp.tohoku.ac.jp

ISSN-1346-9843 doi:10.1253/circj.CJ-13-0269

All rights are reserved to the Japanese Circulation Society. For permissions, please e-mail: cj@j-circ.or.jp 


\section{Methods}

This research is a longitudinal cohort study over 7 years. The participants of this study were type 2 diabetic patients whose physician suspected the existence of severe arteriosclerosis causing RAAS (Supplementary File 1-1A). The primary endpoint of this study is an initial development of CVRE onset (in patients that suffered a CVRE) or the end of the 7-year followup period (in patients that did not experience a CVRE). The influence of the presence/absence of RAAS, a decline in eGFR, clinical factors and surrogate arteriosclerotic markers on patient's CVREs were assessed.

The presence or absence of RAAS was confirmed by magnetic resonance imaging (MRA) using gadolinium contrast media $^{3-6}$ in 200 diabetic out-patients that attended our hospital and who were enrolled into this longitudinal study between January and March 2003 (baseline). The subjects, who were registered into this study, were followed up as outpatients of our hospital or our associated hospital. The number of subjects was established based on a power analysis (Supplementary File 1-1B). Patients whose MRA images showed stenosis of $50 \%$ or more in the renal arteries, ${ }^{7-9}$ as assessed by at least 3 professionals (including a radiologist, cardiologist and nephrologist), were diagnosed with RAAS. A flowchart showing the number of patients included and that of the patients excluded during the follow-up period is provided in Supplementary File 1-1C. Ultimately, a total of 162 patients who met the aforementioned criteria were followed for 7 years, to determine whether they suffered a CVRE in that period.

The CVREs considered in this study consisted of death (from any cause), cerebral infarction, cerebral hemorrhage, transient cerebral ischemia, asymptomatic cerebrovascular disorder, myocardial infarction, angina pectoris, heart failure, initiation of dialysis, a doubling of serum creatinine, leg amputation and the implementation of revascularization in any organ. Only the initial event was counted even if a patient subsequently suffered additional CVREs. In diabetes, there are many cases of asymptomatic CVREs, so, with asymptomatic CVREs whose onset period is unclear, we designated, as the date of onset, the date when the primary physician was able to confirm CVREs (Supplementary File 1-1D). Chest X-rays, ECGs and MRIs of the brain were obtained at baseline for all patients and similar tests were performed when the onset of a CVRE was suspected. These tests were also performed at the end of the study, even in the absence of symptoms, and if a brain MRI revealed any abnormalities in asymptomatic cases, such as an increased size of infarction foci (as assessed by a radiologist), the patients were diagnosed as having suffered an asymptomatic cerebrovascular event. Each CVRE was diagnosed by more than 1 medical specialist from the corresponding area following established diagnostic guidelines. ${ }^{10,11}$ The authors were not involved at any stage of these diagnostic processes.

The presence or absence of RAAS [presented as RAAS (+) and RAAS (-), respectively] was examined by contrast MRA, and the eGFR, age, blood glucose, blood pressure and lipids, as well as the presence or absence of obesity, smoking, left ventricular hypertrophy $(\mathrm{LVH})$ on ECG, and diabetic retinopathies were also evaluated at baseline. In addition, other parameters measured included the intima-media thickness (IMT), brachialankle pulse wave velocity (baPWV), adiponectin, interleukin-6 (IL-6), monocyte-chemoattractant protein (MCP-1), interferoninducible protein (IP-10), vascular endothelial growth factor (VEGF), angiopoietin-2, atrial natriutetic peptide (ANP) and brain natriuretic peptide (BNP), as well as the decline in eGFR from the baseline to the time of CVRE onset (in patients that suffered a CVRE) or until the end of the 7-year follow-up period (in patients that did not experience a CVRE). The influence of these factors on the onset of CVREs during the 7 years was studied, and we assessed if they constituted risk factors for RAAS.

This study received approval from the Ethics Committee at the Tohoku University Hospital, and all patients provided their informed written consent before any tests were performed.

\section{Measurements}

The methods used to measure adiponectin, IL-6, MCP-1, IP-10 and VEGF have been described previously. ${ }^{12}$ Angiopoietin-2 was determined using the commercially available Human Angiopoietin-2 Immunoassay Kit (Human R\&D Systems Inc, MN, USA). The measuring method of $\mathrm{IMT}^{13}$ and baPWV ${ }^{14}$ adopted in this study is shown in Supplementary File 1-1E. The equation for calculating the eGFR is described in Supplementary File 1-1F.

\section{Statistical Analyses}

Numerical values with a normal distribution are expressed as the mean \pm SEM, and those that did not entirely conform to a normal distribution are expressed as the median (range). The t-test was used to perform inter-group comparisons of normally distributed numeric measurements and the Mann-Whitney U-test was used for those measurements that were not distributed normally. The $\chi^{2}$-test was used to compare the prevalence rate of diabetic retinopathy, rate of smoking, CVRE incidence, RAAS prevalence rate and other factors between groups.

In addition, in this longitudinal study, 3 types of analyses were performed: analysis that uses CVREs as the dependent variable and only those factors that could be identified at the baseline as independent variables constitutes a prospective analysis, so a Cox regression analysis was used. In contrast, because analysis that added the percentage change of eGFR to the independent factor becomes a retrospective analysis, a logistic regression analysis was used. A multiple regression analysis was used for items where dependent variables were in numerical form (eg, percentage changes of eGFR and angiopoietin-2 levels, etc.), rather than categories such as "presence or absence of CVREs" (Supplementary File 1-1G).

The method of selecting the factors that should be analyzed as independent factors in the Cox regression analysis is shown in Supplementary File 1-1H.

\section{Results}

The baseline clinical characteristics of all 162 patients were analyzed as a whole and, in addition, these parameters were compared between RAAS $(+)(n=36)$ and RAAS $(-)(n=126)$ patients (Table $\mathbf{S 2}$ ). Compared with the RAAS (-) patients, RAAS (+) patients had lower hemoglobin A1c (HbA1c), eGFR and high-density lipoprotein cholesterol (HDL-C) levels, and higher systolic blood pressure (SBP), heart rate (HR), IMT, baPWV, diabetic retinopathy prevalence and smoking rate, as well as higher adiponectin, IL-6, MCP-1, IP-10, VEGF and angiopoietin-2 levels. With regards to drug administration, the proportion of patients that received each type of treatment was $88.9 \%$ RAAS (+) and $64.3 \%$ RAAS (-) $(\mathrm{P}=0.86)$ for rennin angiotensin system inhibitors (RASIs); $97.2 \%$ and $57.2 \%(\mathrm{P}=0.36)$ for calcium channel blockers (CCBs); $83.3 \%$ and $33.3 \%(\mathrm{P}=0.42)$ for other anti-hypertensive agents; $80.6 \%$ and $38.8 \%(\mathrm{P}=0.37)$ for insulin therapy; $66.7 \%$ and $77.8 \%(\mathrm{P}-0.79)$ for oral hypoglycemic agents; $58.3 \%$ and $42.9 \%(\mathrm{P}=0.89)$ for statins; and $80.6 \%$ and $26.2 \%$ 


\begin{tabular}{|c|c|c|c|c|c|c|}
\hline CVREs & Haz.Ratio & Std.Err. & $\mathbf{Z}$ & $\mathbf{P}$ & \multicolumn{2}{|c|}{ 95\% Conf. Interval } \\
\hline SBP & 1.017796 & 0.0071446 & 2.51 & 0.012 & 1.003889 & 1.03190 \\
\hline IMT & 0.764413 & 0.1772249 & -1.16 & 0.247 & 0.485268 & 1.20413 \\
\hline BNP & 1.003115 & 0.0017999 & 1.73 & 0.083 & 0.999593 & 1.00665 \\
\hline RAAS & 3.526322 & 0.7229337 & 6.15 & 0.000 & 2.359481 & 5.27020 \\
\hline LVH & 1.686646 & 0.5998832 & 1.47 & 0.142 & 0.840002 & 3.38663 \\
\hline
\end{tabular}

SBP, systolic blood pressure; IMT, intima-media thickness; baPWV, brachial-ankle pulse wave velocity; BNP, brain natriuretic peptide; RAAS, renal artery arteriosclerosis; LVH, left ventricular hypertrophy; Haz. Ratio, hazard ratio; Std. Err., standared error; Conf., confidence.

\begin{tabular}{|lcccccc|}
\hline \multicolumn{7}{|c|}{ Table 2. Result of a Cox Regression Analysis Using CVREs as the Dependent Variable, and MCP-1, IP10, } \\
8-OHdG, and AGP2 as the Independent Variables \\
CVREs & Haz.Ratio & Std.Err. & Z & P & $\mathbf{9 5 \%}$ Conf. Interval \\
MCP-1 & 1.00198 & 0.00267 & 0.74 & 0.458 & 0.996759 & 1.007226 \\
IP-10 & 1.00169 & 0.00134 & 1.26 & 0.207 & 0.999069 & 1.004319 \\
8-OHdG & 1.04970 & 0.03117 & 1.63 & 0.102 & 0.990339 & 1.112608 \\
AGP2 & 1.00079 & 0.00011 & 7.01 & 0.000 & 1.000565 & 1.001005 \\
\hline
\end{tabular}

CVRE, cardiovascular-renal events; MCP-1, monocyte chemoattractant protein 1; IP-10, interferon-inducible protein 10; 8-OHdG, urinary 8-hydroxydeoxyguanosine; AGP2, angiopoietin-2. Haz. Ratio, hazard ratio; Std.Err., standared error; Conf., confidence.

\begin{tabular}{|lcccccc|}
\hline \multicolumn{5}{|c|}{ Table 3. Results of a Logistic Regression Analysis that used CVREs as the Dependent Variable and the } \\
Independent Variables, \% change of eGFR, Age, Gender, RAAS and Angiopoietin-2
\end{tabular}

CVREs, cardiovascular-renal events; RAAS, renal artery arteriosclerosis; eGFR, estimated glomerular filtration rate; Std.Err., standard error.

$(\mathrm{P}=0.34)$ for anti-platelet agents (Table $\mathrm{S} 4)$.

CVREs occurred in $24.7 \%$ (40 patients) of all the participants during the 7-year study period. When analyzed according to the presence or absence of RAAS, $75.0 \%$ (27 patients) of RAAS (+) patients and $10.3 \%$ (13 patients) of RAAS $(-)$ patients developed CVREs, reflecting the significantly higher occurrence of these events in RAAS $(+)$ patients $(\mathrm{P}<0.001)$. There were more RAAS $(+)$ patients than RAAS $(-)$ patients when CVREs were considered by type, except for the category "others" (Table S5). Among the RAAS (+) patients, the incidence of CVREs was concentrated in the first half of the observation period ( $1-4$ years), while patients in the RAAS (-) group developed CVREs with equal frequency each year (Table S6).

In terms of function of the development of CVREs during the 7-year follow-up period, the patients were subdivided into 2 groups: CVRE $(-)(n=122)$ and CVRE $(+)(n=40)$. When the baseline clinical characteristics of these groups were compared (Table S3), age, SBP, IMT, baPWV, ANP, BNP, MCP-1, IP10 , angiopoietin-2, presence of LVH and RAAS (+) prevalence were all higher in the CVRE (+) Group, while HbA1c, eGFR and HDL-C levels were lower. RAAS (+) patients accounted for as many as $67.5 \%(26 / 40)$ of the patients in the CVRE (+) Group, whereas only 7.4\% (9/122) of CVRE (-) patients suffered RAAS at baseline $(\mathrm{P}<0.01)$. Furthermore, while the eGFR $\left(\mathrm{ml} \cdot \mathrm{min}^{-1} \cdot 1.73 \mathrm{~m}^{-2}\right)$ decreased by $52.3 \pm 2.3 \%$ in the CVRE (+) patients (from 64.2 \pm 5.1 to $29.6 \pm 2.3 \mathrm{ml} \cdot \mathrm{min}^{-1}$ $\left.\cdot 1.73 \mathrm{~m}^{-2}\right)$, it decreased less in the CVRE (-) patients $\left(16.4 \pm 1.5 \%\right.$ : from $83.6 \pm 2.4$ to $\left.69.7 \pm 2.2 \mathrm{ml} \cdot \mathrm{min}^{-1} \cdot 1.73 \mathrm{~m}^{-2}\right)$. Hence, the CVRE (+) patients had a lower baseline eGFR $(\mathrm{P}<0.001)$ and a greater decline in eGFR $(\mathrm{P}<0.001)$ than those in the CVRE (-) Group.

The rate of RASI use by the RAAS (+) patients was no different from that of the RAAS (-) group, nor were there differences in the \% change in eGFR between RASI monotherapy ("mono") and concomitant therapy ("dual") in both RAAS (+) and RAAS (-) groups. However, both therapies provoked a greater \% decline in eGFR in the RAAS (+) as opposed to the respective therapy in the RAAS $(-)$ patients $(\mathrm{P}<0.01$, Table $\mathrm{S} 7)$. When the patients were subdivided into LVH (+) and LVH (-) groups, the LVH (+) group showed a greater incidence of CVREs, \% decline of eGFR and prevalence of RAAS than the LVH (-) group (Table S8).

There was a lower incidence of CVREs and a smaller decline in eGFR in the RAAS (-) group than in the RAAS (+) group. Moreover, patients with bilateral RAAS had a greater incidence rate of CVREs than those with unilateral RAAS. The changes 

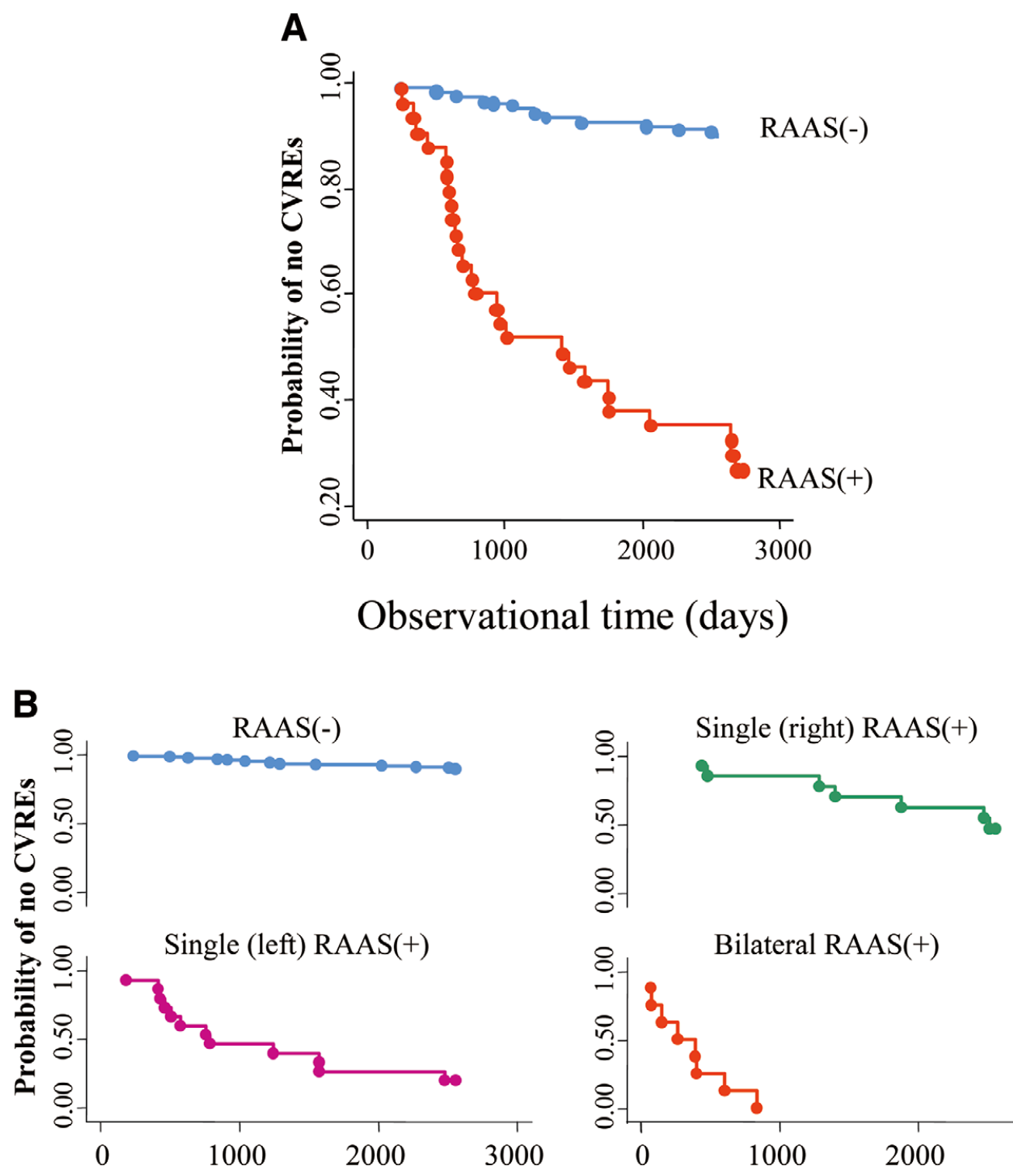

\section{Observational time (days)}

Figure. Probability of not experiencing a CVRE over time. Compared with RAAS $(-)$ patients, the probability of not experiencing CVREs continued to decrease sharply over time in the RAAS (+) patients, with a particularly sharp decrease during the first 1,000 days (A). The probability decreased even in unilateral RAAS patients, although patients with RAAS in both renal arteries (bilateral) experienced an extremely sharp decrease in the probability of not experiencing CVREs, with all the patients having developed CVRE within 1,000 days (B), indicating that bilateral RAAS carries an extremely high risk for CVRE onset. CVRE, cardiovascular-renal events; RAAS, renal artery arteriosclerosis.

in eGFR were not significantly different between the bilateral and unilateral groups (Table S9).

Cox regression analyses were performed using CVREs as the dependent variable, and SBP, IMT, BNP, RAAS and LVH as the independent variables (Supplementary File 1-1H) (Table 1). The factor that influenced CVREs most strongly was RAAS, with a hazard ratio of 3.53 (95\% CI: 2.36-5.27) and $\mathrm{P}<0.001$, followed by SBP with a hazard ratio of $1.02(95 \%$ CI: $1.00-1.03$ ) and $\mathrm{P}=0.012$; these 2 variables were significant independent risk factors for CVREs. By contrast, another Cox regression analysis that used CVREs as the dependent variable, and MCP-1, IP-10, urinary 8-hydroxydeoxyguanosine (8-OHdG), and angiopoietin-2 as the independent variables (Supplementary File 1-1H) only identified angiopoietin-2 to be a significant independent risk factor $(\mathrm{P}<0.001$, Table 2). We therefore conducted a Cox regression analysis, using RAAS and angiopoietin-2 as the independent variables. The results showed that both RAAS and angiopoietin-2 were independent factors for CVREs (Table S10). Of these, RAAS had the largest hazard ratio of 1.84 (95\% CI: 1.18-2.85). 
A logistic regression analysis using the presence or absence of CVREs as a dependent variable identified the percentage change of eGFR $(\mathrm{P}=0.001)$ and angiopoietin-2 $(\mathrm{P}=0.001)$ as independent risk factors, while RAAS was not even close to being a significant factor $(\mathrm{P}=0.678)$. Accordingly, the decline in eGFR was identified as a risk factor, while RAAS no longer represented a risk factor for the occurrence of CVREs (Table 3). This showed that RAAS and the percentage change of eGFR were strongly related (Table S11), and that the percentage change of eGFR was even more strongly related to CVREs (Table 3). Intima-media thickness, which is an indicator for systemic arteriosclerosis, can be considered to be closely related to RAAS (Table S11). Because the systemic vascular disorders and the renal damage progress appears concomitantly in diabetes mellitus, and given that RAAS is directly related to both conditions, it is assumed that RAAS in diabetic patients might serve as a potent predictor of CVREs. Angiopoietin-2 was also identified as an independent risk factor of RAAS (Table S12). However, the percentage change of eGFR and IMT were stronger risk factors of RAAS than angiopoietin-2 (Table S13). Furthermore, eGFR, angiopoietin-2, and RAAS were very closely related each other (Tables S14,S15)

Compared with the RAAS (-) patients, the probability that RAAS (+) patients did not develop a CVRE continued to decrease as the study progressed, decreasing particularly sharply over the first 1,000 days (Figure A). Such decreases were even evident in the unilateral RAAS patients, even though it was less severe than in bilateral RAAS patients (Figure B), indicating that bilateral RAAS $(+)$ patients are at high risk of experiencing a CVRE. The differences of the background between patients who developed CVREs during the first 1,000 days after the observation began, and patients who developed CVREs after the first 1,000 days are shown in Table S16. Twenty-three subjects developed CVREs within 1,000 days (Group A), and 17 developed CVREs after 1,000 days (Group B). Compared with Group B subjects, those in Group A had higher HR, IMT, MCP-1, and angiopoietin-2 values, and lower HDL-C values. Group A also had a greater decline in eGFR than Group B, and Group A had a higher rate of RAAS (+), especially that of bilateral RAAS (+), than Group B. We therefore conducted a logistic regression analysis, using the onset of CVREs within 1,000 days as the dependent variable, and the $\mathrm{HR}$, IMT, and the percentage change of eGFR, as well as MCP-1, angiopoietin-2, HDL-C, and RAAS (+), as the independent variables (Table S17). However, only the percentage change of eGFR was identified as a factor that induced CVREs within 1,000 days, not angiopoietin-2 and RAAS (Table S18).

Fortunately, these results did not change, even after eliminating the 5 patients who developed ESRD and after making an analysis.

\section{Discussion}

Until now, the RAAS (+) lesion itself was believed to be a risk factor for CVREs. ${ }^{15}$ We set up the hypothesis that, under conditions where RAAS is not only caused by RAAS $(+)$ itself but also because of peripheral vascular disorders, intra-renal ischemia would increase, tubulointerstitial disorder has progressed, the level of eGFR would drop, and CVREs would increase as a result. In this longitudinal study, we clarified that RAAS (+) and a decline in eGFR are both independent risk factors for CVREs in diabetes mellitus patients; the latter being more powerful than the former. We then revealed that this decline in eGFR became an even stronger risk factor for CVREs than the presence of RAAS (+) itself, with bilateral RAAS (+) being an extremely strong risk factor. In particular, bilateral RAAS (+) was an extremely strong risk factor for developing CVREs within 1,000 days. However, the greater chance of developing CVREs within 1,000 days seemed to be due to the sharp decline in eGFR of the patient with bilateral RAAS (+). In contrast, angiopoietin-2, which is an ischemic marker, was identified as a risk factor for the decline in eGFR.

All the diabetic patients enrolled on this study were suspected of having RAAS and therefore underwent a MRA, from which only renal arterial stenosis $\geq 50 \%$ was considered as significant arteriosclerosis, ${ }^{3-9}$ irrespective of whether it was clinically relevant stenosis. In fact, there were patients with no changes in RASIs renal perfusion scintigraphy, and some without significant changes in pressure between the renal and aortic sides of the stenotic lesion. Accordingly, we assumed that the eGFR decline detected in our patients with RAAS could be attributed to arteriosclerosis progressing at a more peripheral level than in the renal artery and that it had induced RAAS in these patients, rather than this event being due to the RAAS lesion itself. Although the degree of stenosis in the renal artery is not necessarily related to the progress in the decline in eGFR, ${ }^{16}$ and no differences in changes in renal function were evident between patients with RAAS treated by angioplasty or pharmacologically, ${ }^{8}$ it is thought that the subsequent onset of CVREs in diabetic patients with RAAS is closely related to the low eGFR values. ${ }^{17,18}$ Accordingly, CVREs might occur as a result of the decline in eGFR but not due to the high degree of renal artery stenosis. In other words, in addition to the impaired renal blood flow, provoked by the renal damage directly caused by a reduced renal arterial diameter, diabetic patients with RAAS also suffer impairments in the more peripheral intrarenal microcirculation, both of which would cause more profound ischemic damage to the renal parenchyma and might be reflected in the marked decline in their eGFR. Moreover, the IMT and RAAS (+) were shown to be independent risk factors of the long-term decline in eGFR here, indicating that not only local but also systemic arteriosclerosis is related to a long-term deterioration of renal function. (Table S11) Renal arterial stenotic lesions are probably just one part of the systemic arteriosclerotic lesions. Indeed, RAAS was strongly correlated with cardiovascular markers and RAAS (+) patients frequently developed CVREs as a complication, both here and elsewhere. ${ }^{6,19,20}$

RAAS is also closely related to arteriosclerosis of the coronary artery, ${ }^{21-23}$ as well as to a significant reduction in cardiac function, ${ }^{24}$ which when combined with the reduction in renal function is deemed to make patients prone to heart failure. Indeed, our bilateral RAAS $(+)$ patients were deemed to be especially prone to experience a decline in eGFR, even despite the more aggressive interventions compared to those offered to individuals with unilateral RAAS. Moreover, it appeared that the stronger the decrease in eGFR in a patient, the sooner he/she was likely to suffer a CVRE. Although there were no differences in the incidence of CVREs and in the decline in eGFR between unilateral and bilateral RAAS patients, the time until the onset of CVREs did differ. CVREs are likely to be induced more readily in unilateral RAAS (+) than in RAAS (-) cases, moreover, bilateral RAAS $(+)$ cases were shown to be more susceptible to CVREs, within even a shorter period of time (within ca. 1,000 days). Only the percentage change of eGFR was identified as a factor that induced CVREs within 1,000 days, not angiopoietin-2 and RAAS (Supplementary File 1-7). Therefore, a rapid and significant decline in renal function, rather than ischemia, appears to be closely related to rapidly developing CVREs, and a decline in renal function is a factor that directly triggers CVREs. RAAS can be considered to con- 
stitute a risk that aggravates survival ${ }^{15}$ as it is closely correlated to the decline in eGFR, an index for the progression of renal dysfunction ${ }^{25,26}$ that is more strongly correlated to the onset of CVREs than RAAS itself. This decline in eGFR in diabetic patients with RAAS not only reflects the ischemia in the kidney but might also imply that similar arteriolosclerosis has occurred in other organs (heart, brain and foot etc.), and accordingly, organ ischemia has advanced. Thus, when a patient experiences a rapid decline in eGFR, not only should RAAS be suspected but also, the aggravation of systemic ischemic lesions should be assessed. We thus believe it important to monitor eGFR in daily clinical practice.

In this study, angiopoietin-2 and VEGF were evaluated as markers of ischemia; it is angiopoietin-2 that is more closely related to CVREs. ${ }^{27}$ Angiopoietin-2 is believed to be produced mainly in connection with neovascularization of ischemic lesions. Blood angiopoietin-2 levels rise in line with the decline in renal function and they serve as a predictor of CVREs. ${ }^{28-32}$ Thus, it appears likely that impaired intrarenal blood flow due to arteriosclerosis, as well as renal damage due to renal ischemia caused by sclerotic changes in peripheral vessels (ie, arterioles) rather than by RAAS itself, and the increased angiopoietin-2 levels triggered under these circumstances, might intervene in a complicated manner to cause further cardiovascular/renal disorders. Accordingly, a decline in renal function and elevated angiopoietin-2, as opposed to stenotic lesion itself, might be associated with the heightened incidence of CVREs in type 2 diabetic patients with RAAS (Supplementary File 1-8). By combining the evaluation of albuminuria, which is currently being conducted in diabetes, with the evaluation of the change in eGFR, we can expect to see the advent of higher-precision medical treatment.

One of the limitations of this study is the low number of patients analyzed, with 38 out of the 200 patients having dropped out.

\section{Acknowledgments}

In publishing this study, we wish to express our appreciation to the radiologists, cardiologists and nephrologists who cooperated in interpreting the MRA data, the radiology department staff who assisted with the interpretation of the MRI data, the members of the cardiology department who diagnosed and treated the cardiovascular events, the members of the emergency medicine department who helped transport patients by ambulance, and the hospital staff at both the Tohoku University Hospital and its associated hospital units that assisted the patients. We also thank Takashi Watanabe, M.D. (Division of Epidemiology, Department of Public Health and Forensic Medicine, Tohoku University Graduate School of Medicine) for help with the statistical analyses.

This work was supported by a 21st Century Center of Excellence Program Special Research Grant from the Ministry of Education, Sports and Culture, a research grant for cardiovascular research (13C-5) from the Japanese Ministry of Health, Labor and Welfare, Longitudinal/Cross-Sectional Studies to Generate Evidence for the Diagnosis/Management of Metabolic Syndrome for Health Guidance, and Longitudinal/Cross-Sectional Studies to Generate Evidence for Diagnosis/Management of Metabolic Syndrome in the Governmental Health Check-up and Guidance System.

\section{Disclosure}

None.

\section{References}

1. Saito I. Epidemiological evidence of type 2 diabetes mellitus, metabolic syndrome, and cardiovascular disease in Japan. Circ J 2012; 76: 1066-1073.

2. Manabe I. Chronic inflammation links cardiovascular, metabolic and renal diseases. Circ J 2011; 75: 2739-2748.

3. Duda SH, Schick F, Teufl F, Müller-Schimpfle M, Erley C, Schneider $\mathrm{W}$, et al. Phase-contrast MR angiography for detection of arteriosclerotic renal artery stenosis. Acta Radiol 1997; 38: 287-291.
4. Vasbinder GB, Nelemans PJ, Kessels AG, Kroon AA, de Leeuw PW, van Engelshoven JM. Diagnostic tests for renal artery stenosis in patients suspected of having renovascular hypertension: A metaanalysis. Ann Intern Med 2001; 135: 401-411.

5. Hirsch AT, Haskal ZJ, Hertzer NR, Bakal CW, Creager MA, Halperin JL, et al. ACC/AHA 2005 Practice Guidelines for the management of patients with peripheral arterial disease (lower extremity, renal, mesenteric, and abdominal aortic): A collaborative report from the American Association for Vascular Surgery/Society for Vascular Surgery, Society for Cardiovascular Angiography and Interventions, Society for Vascular Medicine and Biology, Society of Interventional Radiology, and the ACC/AHA Task Force on Practice Guidelines (Writing Committee to Develop Guidelines for the Management of Patients With Peripheral Arterial Disease): Endorsed by the American Association of Cardiovascular and Pulmonary Rehabilitation; National Heart, Lung, and Blood Institute; Society for Vascular Nursing; TransAtlantic Inter-Society Consensus; and Vascular Disease Foundation. Circulation 2006; 113: e463-e654. http://www.ncbi.nlm.nih. gov/pubmed/16549646 (accessed March 21 2006).

6. Dzielińska Z, Januszewicz A, Demkow M, Makowiecka-Cieśla M, Prejbisz A, Naruszewicz M, et al. Cardiovascular risk factors in hypertensive patients with coronary artery disease and coexisting renal artery stenosis. J Hypertens 2007; 25: 663-670.

7. Webster J, Marshall F, Abdalla M, Dominiczak A, Edwards R, Isles $\mathrm{CG}$, et al. Randomised comparison of percutaneous angioplasty vs continued medical therapy for hypertensive patients with atheromatous renal artery stenosis: Scottish and Newcastle Renal Artery Stenosis Collaborative Group. J Hum Hypertens 1998; 12: 329-335.

8. van Jaarsveld BC, Krijnen P, Pieterman H, Derkx FH, Deinum J, Postma CT, et al. The effect of balloon angioplasty on hypertension in atherosclerotic renal-artery stenosis: Dutch Renal Artery Stenosis Intervention Cooperative Study Group. N Engl J Med 2000; 342: $1007-1014$.

9. Tanemoto M, Saitoh H, Satoh F, Satoh H, Abe T, Ito S. Predictors of undiagnosed renal artery stenosis among Japanese patients with risk factors of atherosclerosis. Hypertens Res 2005; 28: 237-242.

10. Pearce JD, Craven BL, Craven TE, Piercy KT, Stafford JM, Edwards MS, et al. Progression of atherosclerotic renovascular disease: A prospective population-based study. J Vasc Surg 2006; 44: 955-962.

11. Cheung CM, Wright JR, Shurrab AE, Mamtora H, Foley RN, O'Donoghue DJ, et al. Epidemiology of renal dysfunction and patient outcome in atherosclerotic renal artery occlusion. J Am Soc Nephrol 2002; 13: 149-157.

12. Wright JR, Shurrab AE, Cheung C, Waldek S, O'Donoghue DJ, Foley $\mathrm{RN}$, et al. A prospective study of the determinants of renal functional outcome and mortality in atherosclerotic renovascular disease. Am J Kidney Dis 2002; 39: 1153-1161.

13. Cohen MG, Pascua JA, Garcia-Ben M, Rojas-Matas CA, Gabay JM, Berrocal DH, et al. A simple prediction rule for significant renal artery stenosis in patients undergoing cardiac catheterization. Am Heart J 2005; 150: $1204-1211$.

14. Nakamura S, Iihara K, Matayoshi T, Yasuda H, Yoshihara F, Kamide $\mathrm{K}$, et al. The incidence and risk factors of renal artery stenosis in patients with severe carotid artery stenosis. Hypertens Res 2007; 30: 839-844.

15. Patel JV, Lim HS, Varughese GI, Hughes EA, Lip GY. Angiopoietin2 levels as a biomarker of cardiovascular risk in patients with hypertension. Ann Med 2008; 40: 215-222.

16. Harding MB, Smith LR, Himmelstein SI, Harrison K, Phillips HR, Schwab SJ, et al. Renal artery stenosis: Prevalence and associated risk factors in patients undergoing routine cardiac catheterization. $J \mathrm{Am}$ Soc Nephrol 1992; 2: 1608-1616.

17. Uzu T, Inoue T, Fujii T, Nakamura S, Inenaga T, Yutani C, et al. Prevalence and predictors of renal artery stenosis in patients with myocardial infarction. Am J Kidney Dis 1997; 29: 733-738.

18. Yamashita T, Ito F, Iwakiri N, Mitsuyama H, Fujii S, Kitabatake A. Prevalence and predictors of renal artery stenosis in patients undergoing cardiac catheterization. Hypertens Res 2002; 25: 553-557.

19. Wright JR, Shurrab AE, Cooper A, Kalra PR, Foley RN, Kalra PA. Progression of cardiac dysfunction in patients with atherosclerotic renovascular disease. QJM 2009; 102: 695-704.

20. Uzu T, Takeji M, Yamada N, Fujii T, Yamauchi A, Takishita S, et al. Prevalence and outcome of renal artery stenosis in atherosclerotic patients with renal dysfunction. Hypertens Res 2002; 25: 537-542.

21. Caps MT, Perissinotto C, Zierler RE, Polissar NL, Bergelin RO, Tullis $\mathrm{MJ}$, et al. Prospective study of atherosclerotic disease progression in the renal artery. Circulation 1998; 98: 2866-2872.

22. Caps MT, Zierler RE, Polissar NL, Bergelin RO, Beach KW, Cantwell-Gab K, et al. Risk of atrophy in kidneys with atherosclerotic renal artery stenosis. Kidney Int 1998; 53: 735-742. 
23. Lim HS, Blann AD, Chong AY, Freestone B, Lip GY. Plasma vascular endothelial growth factor, angiopoietin-1, and angiopoietin-2 in diabetes: Implications for cardiovascular risk and effects of multifactorial intervention. Diabetes Care 2004; 27: 2918-2924.

24. David S, John SG, Jefferies HJ, Sigrist MK, Kümpers P, Kielstein JT, et al. Angiopoietin-2 levels predict mortality in CKD patients. Nephrol Dial Transplant 2012; 27: 1867-1872.

25. Golledge J, Clancy P, Yeap BB, Hankey GJ, Norman PE. Increased serum angiopoietin-2 is associated with abdominal aortic aneurysm prevalence and cardiovascular mortality in older men. Int J Cardiol 2012 April 4, doi:10.1016/j.ijcard.2012.03.120 [Epub ahead of print].

26. Chen J, Yu H, Sun K, Song W, Bai Y, Yang T, et al. Promoter variant of angiopoietin-2 and plasma angiopoietin-2 are associated with risk of stroke recurrence in lacunar infarct patients. Biochem Biophys Res Commun 2010; 398: 212-216.

27. Nadar SK, Blann A, Beevers DG, Lip GY. Abnormal angiopoietins $1 \& 2$, angiopoietin receptor Tie-2 and vascular endothelial growth factor levels in hypertension: Relationship to target organ damage [a sub-study of the Anglo-Scandinavian Cardiac Outcomes Trial (ASCOT)]. J Intern Med 2005; 258: 336-343.

28. Apple FS, Wu AH, Jaffe AS. European Society of Cardiology and American College of Cardiology guidelines for redefinition of myocardial infarction: How to use existing assays clinically and for clinical trials. Am Heart J 2002; 144: $981-986$.

29. Crespi V, Braga M, Beretta S, Carolei A, Bignamini A, Sacco S. A practical definition of minor stroke. Neurol Sci 2013; 34: 1083-1086.

30. Ogawa S, Nakayama K, Nakayama M, Mori T, Matsushima M, Okamura M, et al. Methylglyoxal is a predictor in type 2 diabetic patients of intima-media thickening and elevation of blood pressure. Hypertension 2010; 56: 471-476.

31. Temelkova-Kurktschiev TS, Koehler C, Leonhardt W, Schaper F, Henkel E, Siegert G, et al. Increased intimal-medial thickness in newly detected type 2 diabetes: Risk factors. Diabetes Care 1999; 22: 333 -
338.

32. Yamashina A, Tomiyama H, Arai T, Hirose K, Koji Y, Hirayama Y, et al. Brachial-ankle pulse wave velocity as a marker of atherosclerotic vascular damage and cardiovascular risk. Hypertens Res 2003; 26: $615-622$.

\section{Supplementary Files}

Supplementary File 1

1. Preparation for the study and passage of the study

2. Clinical characteristics of the study subjects, and the comparisons between the RAAS (+) and RAAS (-) groups at baseline or CVREs $(+)$ and CVREs (-) during the 7-year follow up

3. Differences in the incidence of CVREs in the RAAS (+) and the RAAS (-) groups

4. Result of a cox regression analysis that used CVREs as the dependent variable

5. Results of logistic regression analyses using RAAS as a dependent variable

6. Results of multiple regression analyses

7. Baseline characteristics of the subjects who developed CVREs during the first 1,000 days and the independent risk factors that used CVREs were developed during the first 1,000 days

8. Relationship and the pathophysiology of the renal artery injury and CVREs

Please find supplementary file(s);

http://dx.doi.org/10.1253/circj.CJ-13-0269 